\title{
Caracterização da estrutura da vegetação numa pastagem natural do Bioma Pampa submetida a diferentes estratégias de manejo da oferta de forragem ${ }^{1}$
}

\author{
Fabio Pereira Neves ${ }^{2}$, Paulo César de Faccio Carvalho ${ }^{3}$, Carlos Nabinger ${ }^{4}$, Igor Justin \\ Carassai $^{5}$, Davi Teixeira dos Santos ${ }^{6}$, Gustavo Velasquez da Veiga ${ }^{7}$
}

\author{
${ }_{1}$ Pesquisa financiada pela Universidade Federal do Rio Grande do Sul (UFRGS) e bolsa de mestrado (CNPq). \\ 2 Doutorando do PPG - Zootecnia - UFRGS. Av. Bento Gonçalves, 7712. Porto Alegre, RS, Brasil. CEP: 91501-970. \\ ${ }^{3}$ Departamento de Plantas Forrageiras e Agrometeorologia, UFRGS. \\ ${ }^{4}$ Departamento de Plantas Forrageiras e Agrometeorologia, UFRGS. \\ ${ }^{5}$ Doutorando do PPG - Zootecnia - UFRGS, bolsista CAPES. \\ ${ }^{6}$ Pós-doutorando do Departamento de Plantas Forrageiras e Agrometeorologia, UFRGS. \\ ${ }^{7}$ Graduação da Faculdade de Agronomia UFRGS, Bolsista de Extensão-PROREXT.
}

RESUMO - Os objetivos neste trabalho foram descrever e investigar a dinâmica espaço-temporal de sítios alimentares em diferentes faixas de massa de forragem e altura do pasto, assim como o percentual de área efetivamente pastejada, a taxa de acúmulo de forragem e a produção de matéria seca de uma pastagem natural do Bioma Pampa. O delineamento utilizado foi o de blocos ao acaso com duas repetições, com três ofertas de forragem fixas $(8,12$ e $16 \%)$ e três ofertas de forragem variáveis ao longo do ano $(8-12 \%, 12-8 \%$ e $16-12 \%)$, com o primeiro valor correspondendo à primavera. Na oferta fixa de forragem de $8 \%$, observou-se maior percentual de área efetivamente pastejada, porém os valores médios de altura e massa de forragem foram inferiores aos das demais ofertas. As maiores ofertas de forragem, 16 e 16-12\%, apresentaram altura média do pasto superior, $9,0 \mathrm{~cm}$ e massa de forragem de $2.000 \mathrm{~kg} /$ ha de matéria seca, porém, com área efetivamente pastejada inferior às observadas nas ofertas de 8 e $8-12 \%$. Mesmo estratégias de manejo tão distintas, aproximadamente 60 a $70 \%$ dos sítios alimentares ocorreram em faixas consideradas limitantes ao potencial de ingestão de forragem por bovinos, com exceção dos tratamentos $16 \%$ e 16-12\%, nos quais se observou menor frequência de sítios alimentares na faixa inferior a $6,0 \mathrm{~cm}$ de altura. Os sítios alimentares, em geral, se concentraram em faixas com altura $<6 \mathrm{~cm}$ e massas de forragem $<1000$ e $1000-1500 \mathrm{~kg} / \mathrm{ha}$ de matéria seca em todas as ofertas de forragem avaliadas, porém, nas ofertas de $16-12 \%$ e $8-12 \%$, ocorreu uma melhor distribuição dos sítios alimentares nas demais faixas de massa de forragem e altura do pasto.

Palavras-chave: altura do pasto, estrutura do pasto, intensidade de pastejo, massa de forragem, sítios alimentares

\section{Structural characterization of a natural pasture vegetation from Pampa Biome under different herbage allowance management strategies}

ABSTRACT - The objective of this trial was to investigate the spatio-temporal dynamics of feeding sites stratified by strips of herbage mass and sward height, and the percentage of effectively grazed area, herbage growth rate and dry matter yield of a natural pasture from Pampa Biome managed with different herbage allowance management strategies. A randomized block design with two replications was used with three fixed herbage allowance $(8,12$ and $16 \%)$ and three herbage allowance variables throughout the year $(8-12 \%, 12-8 \%$, and $16-12 \%)$, with first value corresponding to the spring season. The $8 \%$ fixed herbage allowance presented greater effectively grazed area, but the average height and herbage mass were lower than in other allowances. The highest herbage allowance, $16 \%$ and $16-12 \%$, presented $9.0 \mathrm{~cm}$ average sward height and herbage mass of $2,000 \mathrm{~kg} / \mathrm{ha}$ dry matter, and a smaller effectively grazed area than the $8 \%$ and $8-12 \%$ herbage allowances. In spite of the different management strategies used, between 60 and $70 \%$ of feeding sites occurred in strips considered to constrain animal intake, except in the $16 \%$ and $16-12 \%$ treatments, in which a smaller frequency of feeding sites in strips lower than $6.0 \mathrm{~cm}$ was registered. In general, the feeding sites were concentrated on strips $<6 \mathrm{~cm} \mathrm{SH}$, and herbage masses $<1000$ and $1000-1500 \mathrm{~kg} / \mathrm{ha}$ dry matter, in all allowances. However, at $16-12 \%$ and $8-12 \%$ herbage allowances, the feeding sites were better distributed among the strips for herbage mass and sward height.

Key Words: feeding sites, grazing intensity, herbage mass, sward height, sward structure 


\section{Introdução}

O conceito de oferta de forragem contribuiu para o avanço da pesquisa em manejo de pastagens, pois experimentos com animais em pastejo passaram a ser comparáveis e, nesta nova perspectiva, as taxas de lotação se tornaram reflexo da oferta de recursos tróficos e da capacidade da comunidade vegetal em capturá-los (Carvalho et al., 2007).

Amplitudes de ofertas de forragem de 4\% a 16\% do peso vivo (PV) em pastagem natural proporcionam variações acentuadas na estrutura do pasto (Maraschin, 2001). O aumento na oferta de forragem melhora a participação de espécies e componentes morfológicos em estruturas indesejáveis ao animal. Entretanto, o padrão de desfolhação aumenta a heterogeneidade espacial da vegetação com a formação de uma estrutura do tipo mosaico (Corrêa \& Maraschin, 1994).

Existem dúvidas acerca da formação estrutural da vegetação em ambientes pastoris complexos e da forma como o comportamento ingestivo e a composição da dieta são afetados (Pinto et al., 2007; Gonçalves, 2007). Entretanto, a qualidade da forragem do estrato efetivamente pastejado não é afetada pela oferta de forragem (Soares, 2002). Assim, a limitação para o desempenho animal não seria a concentração em nutrientes da forragem, mas sim a quantidade total de forragem que os animais conseguiriam colher e ingerir (Carvalho et al., 2007).

Em pastagens monoespecíficas, são observadas altas correlações entre a altura do pasto e o comportamento ingestivo. Boval et al. (2007) verificaram correlações de 0,$91 ; 0,79 \mathrm{e}-0,68 \mathrm{da}$ altura do pasto com a profundidade, a massa e a taxa dos bocados, respectivamente. Mesmo em pastagens naturais heterogêneas, a estrutura do pasto afeta as dimensões do bocado. Segundo Gonçalves (2007), a altura do estrato efetivamente pastejado que maximiza a massa do bocado e a taxa de ingestão é de $11,4 \mathrm{~cm}$, que corresponde a massa de forragem de 2.000 a $2.500 \mathrm{~kg} / \mathrm{ha}$ de MS.

Sob a hipótese de que o manejo da oferta de forragem em pastagem natural modifica consideravelmente a estrutura do estrato efetivamente pastejado, este trabalho foi realizado com o objetivo de avaliar a estrutura da vegetação de uma pastagem natural do Bioma Pampa submetida a diferentes estratégias de manejo da oferta de forragem por meio da estratificação de pontos amostrais de massa de forragem e altura do pasto, denominados sítios alimentares, assim como o percentual de área efetivamente pastejada e a produção de forragem nessas estratégias de manejo.

\section{Material e Métodos}

O experimento foi conduzido em uma área de 52 ha de pastagem natural na Estação Experimental Agronômica, pertencente à Universidade Federal do Rio Grande do Sul (EEA/UFRGS), localizada no município de Eldorado do Sul, região da Depressão Central do estado do Rio Grande do Sul. As coordenadas geográficas aproximadas da área experimental são $30^{\circ} 05^{\prime} 27^{\prime \prime} \mathrm{S}, 51^{\circ} 40^{\prime} 18^{\prime \prime} \mathrm{W}$ e $46 \mathrm{~m}$ de altitude. O clima da região é do tipo $\mathrm{Cfa}$, subtropical úmido com verão quente, segundo classificação de Köppen, e a precipitação total média anual, de $1.440 \mathrm{~mm}$ (Bergamaschi et al., 2003). As temperaturas médias mensais variam de 9 a $25^{\circ} \mathrm{C}$ e a média diária de radiação solar global, de 200 a $500 \mathrm{cal} / \mathrm{cm}^{2}$. A composição florística na área experimental foi descrita em levantamentos realizados anteriormente por Escosteguy (1990), Moojen (1991), Boldrini (1993) e indicam vegetação representativa dos campos naturais da região da Depressão Central do Rio Grande do Sul.

Os manejos com níveis fixos de ofertas de forragem são empregados na área desde 1986, pelo método de pastejo contínuo com taxa de lotação variável. A partir do ano 2000, foram adicionadas outras formas de manejo, com alterações anuais na oferta de forragem durante a primavera. O período experimental foi de 388 dias, entre 21 de janeiro de 2006 e 13 de fevereiro de 2007, e os tratamentos consistiram de três níveis fixos e diários de oferta de forragem ( $\mathrm{kg}$ de matéria seca $[\mathrm{MS}] / 100 \mathrm{~kg}$ de peso vivo [PV]; $\% \mathrm{PV})-8 \%, 12 \%$ ou $16 \%$ do PV durante todo o ano - e de três combinações dos níveis de oferta de forragem - $8 \% \mathrm{PV}$ na primavera e $12 \% \mathrm{PV}$ no verão/outono/inverno; $12 \% \mathrm{PV}$ na primavera e $8 \% \mathrm{PV}$ no verão/outono/inverno; e $16 \% \mathrm{PV}$ na primavera e $12 \% \mathrm{PV}$ no verão/outono/inverno.

Foram utilizadas novilhas de corte mestiças em fase de recria, oriundas de cruzamentos entre as raças Angus, Hereford e Nelore, com média de 15 meses de idade e peso vivo (PV) médio inicial de $188 \mathrm{~kg}$. Os procedimentos de pesagem dos animais e as avaliações feitas no pasto foram sempre realizados em intervalos de 28 dias. A área experimental foi mantida sem animais por aproximadamente 70 dias até o início do trabalho.

Para a estimativa da massa de forragem(MF, $\mathrm{kg} / \mathrm{hade} \mathrm{MS})$, foi utilizada a técnica da dupla amostragem, descrita por Wilm et al. (1944). As amostragens foram realizadas aleatoriamente em 50 pontos por unidade experimental, com auxílio de um quadro de ferro de $0,25 \mathrm{~m}^{2}$, exclusivamente no estrato inferior do pasto, excluindo as touceiras compostas por espécies indesejáveis, conforme definições apresentadas por Fontoura Jr. et al. (2007). A decisão de utilizar somente o estrato inferior para compor a massa foi baseada no 
trabalho realizado por Pinto et al. (2007), que encontraram baixa correlação entre o tempo de pastejo e a massa de forragem total (incluindo touceiras na avaliação da massa de forragem) e, ao mesmo tempo, alta correlação com a massa de forragem exclusivamente no estrato inferior.

Foram cortados quatro pontos por unidade experimental, aproveitando os pontos de cortes de gaiola, acima do mantilho. A forragem cortada foi recolhida em sacos de papel, seca em estufa com circulação forçada a $65^{\circ} \mathrm{C}$ por 72 horas e pesada em balança de precisão. Os valores dos cortes foram utilizados para ajuste das estimativas visuais da massa de forragem em cada avaliação por meio de uma regressão linear entre a massa de forragem atribuída pelo avaliador e a massa de forragem cortada.

Durante caminhamento para estimativas visuais de massa de forragem, a frequência de touceiras de espécies e/ou estruturas pré-definidas como indesejáveis foi registrada, de modo que a participação percentual de touceiras nos 50 pontos amostrados foi descontada da área total para estimativa da área efetivamente pastejada ( $\%$ do total da área), comumente chamada estrato inferior ou entre touceiras.

No centro dos quadros amostrados para estimativa da massa de forragem, procedeu-se à medição da altura do pasto $(\mathrm{cm})$ com auxílio de um bastão graduado, segundo método proposto por Barthram (1985). O tamanho das unidades experimental (piquetes) variou entre 3 e 5 ha. A taxa de acúmulo diária de matéria seca ( $\mathrm{kg} / \mathrm{ha} \mathrm{de} \mathrm{MS/dia)} \mathrm{foi}$ medida com o uso de quatro gaiolas de exclusão ao pastejo por unidade experimental, empregando-se a técnica do triplo emparelhamento (Moraes et al., 1990). A produção total de matéria seca foi calculada pelo somatório das produções de cada período, obtidas multiplicando-se a taxa de acúmulo diário pelo número de dias do período.

A disponibilidade diária de forragem ( $\mathrm{kg} / \mathrm{ha} \mathrm{de} \mathrm{MS)} \mathrm{foi}$ calculada pelo quociente entre a massa de forragem inicial menos a massa de forragem final e o número de dias de cada subperíodo experimental e acrescida da taxa de acúmulo diário correspondente ao período. A oferta de forragem real (\% PV) foi obtida dividindo-se a disponibilidade diária de forragem pela taxa de lotação média de cada subperíodo, em $\mathrm{kg} / \mathrm{ha}$ de PV, de modo que o valor obtido foi multiplicado por 100 para expressar a oferta de forragem em porcentagem do peso vivo (\% PV).

Para caracterização estrutural da vegetação, calculou-se o percentual de sítios alimentares em faixas de massa de forragem (MF) e altura (ALT), com base nos resultados de estudos realizados por Gonçalves (2007) e Santos (2007), resultando nas seguintes estratificações: ALT $\leq 6 \mathrm{~cm} \mathrm{e}$ $\mathrm{MF} \leq 1.000 \mathrm{~kg} / \mathrm{ha}$ de MS; ALT entre 6 e $8 \mathrm{~cm}$ e MF 1.000 e $1.500 \mathrm{~kg} / \mathrm{ha}$ de MS; ALT entre 8 e $10 \mathrm{~cm}$ e MF entre $1.500 \mathrm{e}$
$2.000 \mathrm{~kg} / \mathrm{ha}$ de MS; ALT entre 10 e $12 \mathrm{~cm}$ e MF entre 2.000 e $2.500 \mathrm{~kg} / \mathrm{ha}$ de MS; ALT $\geq 12 \mathrm{~cm}$ e $\mathrm{MF} \geq 2.500 \mathrm{~kg} / \mathrm{ha}$ de MS.

De acordo com os resultados obtidos por Gonçalves (2007), pode-se considerar a faixa 10-12 cme $2.000-2.500 \mathrm{~kg} / \mathrm{ha}$ de MS como ótima à ingestão de MS e as faixas $<8 \mathrm{~cm}$ e $1.500 \mathrm{~kg} / \mathrm{ha} \mathrm{de} \mathrm{MS} \mathrm{e}>12 \mathrm{~cm}$ e $2.500 \mathrm{~kg} / \mathrm{ha}$ de MS como faixas que estariam limitando a ingestão de MS. A faixa de $8-10 \mathrm{~cm}$ e $1.500-2.000 \mathrm{~kg} / \mathrm{ha}$ de MS foi considerada não-limitante ao desempenho dos animais, ou seja, na qual o tempo de pastejo pode compensar algumas limitações em estrutura. Para o cálculo dos sítios alimentares, utilizaram-se planilhas do Microsoft Office Excel (versão 2003) e, pela função CONT.SE, calculou-se o percentual de quadros em cada faixa de massa de forragem e altura, caracterizado como sítio alimentar.

O delineamento experimental foi o de blocos completos casualizados com duas repetições, totalizando 12 unidades experimentais, considerando o tipo de solo como critério de bloqueamento. Foram realizadas análises de medidas repetidas no tempo e os dados foram submetidos à análise de variância. Para a escolha da matriz de variância e covariância, utilizou-se o critério de informação Akaike (Wolfinger, 1993). Análise de covariância foi realizada para isolar o efeito de diferenças de percentuais de relevo (áreas de baixada, encosta e topo) entre as unidades experimentais. As médias dos tratamentos foram estimadas utilizando-se o LSMEANS e a comparação entre elas, pelo teste $t$ de Student, a 10\% de significância. Também foram realizadas análises de correlação linear entre as variáveis estudadas.

O modelo estatístico geral referente à análise das variáveis estudadas foi representado por:

$\mathrm{Y}_{\mathrm{ijkl}}=\mu+\beta_{\mathrm{i}}+\mathrm{T}_{\mathrm{j}}+(\beta \mathrm{T})_{\mathrm{ij}}+\gamma_{\mathrm{k}}+(\mathrm{T} \gamma)_{\mathrm{jk}}+\varphi_{1}+\varepsilon_{\mathrm{ijkl}}$

em que: $\mathrm{Y}_{\mathrm{ijkl}}=$ variáveis-respostas; $\mu=$ média inerente a todas as observações; $\beta_{\mathrm{i}}=$ efeito do i-ésimo bloco; $\mathrm{T}_{\mathrm{j}}=$ efeito do j-ésimo tratamento (oferta de forragem); $(\beta T)_{i j}=$ efeito aleatório decorrente da interação i-ésimo bloco $\times$ j-ésimo tratamento (erro a); $\gamma_{k}=$ efeito do k-ésimo tempo observado (estação do ano); $(\mathrm{T} \gamma)_{\mathrm{jk}}=$ efeito da interação j-ésimo tratamento $\times$ k-ésimo tempo; $\varphi_{1}=$ efeito do relevo ajustado como covariável; e $\varepsilon_{\mathrm{ijkl}}=$ erro aleatório, suposto independente e normalmente distribuído (erro b).

Para a comparação entre faixas, o modelo estatístico foi representado por:

$\mathrm{Y}_{\mathrm{ijkl}}=\mu+\beta_{\mathrm{i}}+\mathrm{T}_{\mathrm{j}}+(\beta \mathrm{T})_{\mathrm{ij}}+\gamma_{\mathrm{k}}+(\mathrm{T} \gamma)_{\mathrm{jk}}+\varphi_{1}+(\mathrm{T} \varphi)_{\mathrm{j} 1}+$ $(\beta \mathrm{T} \varphi)_{\mathrm{ijl}}+(\gamma \varphi)_{\mathrm{kl}}+(\mathrm{T} \gamma \varphi)_{\mathrm{jkl}}+\varepsilon_{\mathrm{ijkl}}$

em que: $Y_{i j k l}=$ variáveis-respostas; $\mu=$ média inerente a todas as observações; $\beta_{\mathrm{i}}=$ efeito do i-ésimo bloco; $\mathrm{T}_{\mathrm{j}}=$ efeito do j-ésimo tratamento (oferta de forragem); $(\beta \mathrm{T})_{\mathrm{ij}}=$ efeito aleatório da interação i-ésimo bloco $\times$ j-ésimo 
tratamento (erro a); $\gamma_{k}=$ efeito do k-ésimo tempo observado (estação do ano); $(\mathrm{T} \gamma)_{\mathrm{jk}}=$ efeito da interação j-ésimo tratamento $\times$ k-ésimo tempo; $\varphi_{1}=$ efeito da l-ésima faixa observada; $(\mathrm{T} \varphi)_{\mathrm{j} 1}=$ efeito da interação j-ésimo tratamento $\times$ 1-ésima faixa; $(\beta T \varphi)_{\mathrm{ijl}}=$ efeito aleatório atribuído à interação i-ésimo bloco $\times \mathrm{j}$-ésimo tratamento $\times 1$-ésima faixa $\left(\right.$ errob); $(\gamma \varphi)_{\mathrm{kl}}=$ efeito da interação $\times \mathrm{k}$-ésimo tempo $\times 1$-ésima faixa; $(\mathrm{T} \gamma \varphi)_{\mathrm{jkl}}=$ efeito da interação $\mathrm{j}$-ésimo tratamento $\times$ k-ésimo tempo $\times 1$-ésima faixa; e $\varepsilon_{\mathrm{ijkl}}=$ erro aleatório, suposto independente e normalmente distribuído (erro c).

As análises foram realizadas utilizando-se o pacote estatístico SAS, versão 8.2 (SAS, 2001).

\section{Resultados e Discussão}

$\mathrm{Na}$ média do período experimental, as ofertas de forragem reais diferiram $(\mathrm{P}=0,0116)$ e apresentaram valores próximos às ofertas pretendidas (Tabela 1), resultando em um gradiente entre os níveis e as combinações de oferta de forragem, tal como desejado para testar a hipótese deste trabalho. Não houve interação nível de oferta de forragem $\times$ estação do ano para massa de forragem $(\mathrm{P}=0,9932)$, altura do pasto $(\mathrm{P}=0,9051)$, taxa de acúmulo de forragem $(\mathrm{P}=0,2291)$, área efetivamente pastejada $(\mathrm{P}=0,9574)$, oferta real de forragem $(\mathrm{P}=0,9282)$ e produção de matéria seca $(\mathrm{P}=0,1827)$.

Não se observou efeito $(\mathrm{P}>0,1)$ da oferta de forragem sobre a taxa de acúmulo de forragem nem sobre a produção de matéria seca, que variaram entre 3.060 e $5.000 \mathrm{~kg}$ de MS, respectivamente, para as ofertas de forragem de $12 \%$ e 8-12\%. Nessas pastagens naturais, utilizadas extensivamente e com limitado melhoramento, as taxas de acúmulo de forragem e produção de matéria seca estão principalmente associadas à composição botânica e estrutural da vegetação e são determinadas, em parte, pelas características de solo, temperatura, regime hídrico, topografia, estação do ano e manejo do pastejo (Pallarés et al., 2005).

Em valores absolutos, a produção de matéria seca e a taxa de acúmulo de forragem foram mais altas quando se diminuiu a oferta de forragem para $8 \%$ na primavera $(8-12 \%)$, como reportado em outros trabalhos. Soares et al. (2005) encontraram maior taxa de acúmulo de forragem no verão para oferta de forragem de $8-12 \%(\mathrm{P}<0,1)$, que não diferiu da oferta de $8 \%$. Esse mesmo nível de oferta de forragem foi o que resultou no maior ganho médio diário dos bovinos no período do inverno, atribuído à maior qualidade da forragem ao início dessa estação. Os autores concluíram que a variação estacional da oferta de forragem, particularmente o aumento da oferta após a primavera, tem impactos positivos sobre a produção primária e secundária. Armstrong et al. (1995) também demonstraram que, não só o nível de oferta de forragem atual, mas também o manejo e a condição anteriores do pasto, definem, em parte, a produção animal.

Houve correlação positiva, de 0,87 , entre a massa de forragem e altura do pasto $(\mathrm{P}<0,0001)$. Altas correlações são normalmente encontradas em pastagens cultivadas, com características estruturais mais homogêneas (Pedreira, 2002). Em ambientes de elevada heterogeneidade espacial e temporal, como as pastagens naturais, não é rara a obtenção de baixo grau de correlação entre essas variáveis. Neste experimento, as avaliações se concentraram no estrato efetivamente pastejável (entre touceiras), onde o pasto, em tese, apresenta menor heterogeneidade estrutural, o que provavelmente resultou na elevada correlação observada. Em trabalhos realizados por Santos et al. (2004), Gonçalves (2007) e Carassai et al. (2008) em pastagem natural com estratégias de manejo que proporcionam maior homogeneidade estrutural do pasto, como roçadas, adubação do pasto e manejo da altura, também foram encontrados elevados coeficientes de correlação entre a altura e massa de forragem $(\mathrm{r}=0,70 ; 0,86$ e 0,87 , respectivamente).

$\mathrm{Na}$ média do ano, observou-se que nas ofertas de forragem de $12 \%, 16 \%$ e $16-12 \%$, houve superioridade tanto para massa de forragem ( $\mathrm{P}=0,0032), 2000 \mathrm{~kg} / \mathrm{ha}$ de MS, quanto para altura do pasto $(\mathrm{P}=0,0354), 9,5 \mathrm{~cm}$, em comparação às ofertas de $8 \%$ e $12-8 \%$. Gonçalves (2007) observou que a velocidade de ingestão de novilhas, em pastos naturais semelhantes, somente aumentou em massa de forragem e altura do pasto superiores às médias registradas neste trabalho, indicando que valores de massa de forragem entre 2.000 e $2.500 \mathrm{~kg} / \mathrm{ha}$ de MS e altura entre 10 e $12 \mathrm{~cm}$ podem ser considerados ótimos para melhorar a taxa de ingestão de forragem por bovinos.

O percentual de área efetivamente pastejada (Tabela 1) representa a fração de área composta pelo estrato inferior e onde se concentram os sítios alimentares preferidos (Stuth, 1991). A fração restante corresponde à área ocupada, sobretudo por espécies indesejáveis e/ou por estruturas menos utilizadas pelos animais. Com o aumento da oferta de forragem e da oportunidade de seleção de forragem pelos animais, também aumentam os percentuais de locais não preferidos e/ou menos utilizados pelos animais e que, posteriormente, irão formar estruturas em forma de touceiras, implicando redução da área efetivamente pastejada, que diminuiu de $76 \%$ na oferta de forragem $8 \%$ para $63 \%$ na oferta de forragem de 16\%. De acordo com Santos (2007), a diminuição da área efetivamente pastejada também foi marcante entre as ofertas de forragem de $8 \%$ e $12 \%(80,9$ e $68 \%$, respectivamente).

Neste estudo, a menor oferta de forragem foi de $8 \%$ fixa o ano todo e apresentou o maior percentual de área 
efetivamente pastejada $(\mathrm{P}=0,0705)$ (Tabela 1$)$, porém os valores de massa de forragem e altura no estrato inferior foram os menores $(\mathrm{P}=0,032, \mathrm{P}=0,0354$, respectivamente $)$. A oferta de forragem de $12 \%$ não diferiu $(\mathrm{P}>0,1)$ daquelas de $16 \%$ e $16-12 \%$ em área efetivamente pastejada e, a partir deste nível, os valores parecem estabilizar-se em patamares semelhantes. $\mathrm{Na}$ oferta de forragem de $8-12 \%$, a área efetivamente pastejada manteve-se igual à da oferta de forragem de $8 \%$ fixa, porém com maior altura e massa de forragem $(\mathrm{P}<0,1)$. Essa estratégia parece ser positiva por manter percentual de área efetivamente pastejada relativamente alto, sem alteração na massa de forragem e altura do pasto no estrato efetivamente pastejado.

Existem evidências de que diversos níveis de consumo podem ser obtidos com mesma massa de forragem ou altura (Poppi et al., 1987; Carvalho, 1997). Laca \& Dement (1991) demonstraram que os animais, em grande parte das situações, pastejam sítios onde a massa de forragem e a altura são superiores à média da pastagem. Esses autores demonstraram que, em níveis intermediários de oferta de forragem, a massa de forragem nos sítios alimentares selecionados chega a ser $65 \%$ maior que a média da massa de forragem presente na pastagem. Entretanto, quando a massa de forragem é baixa, o pasto se torna mais homogêneo e diminuem as diferenças em seleção entre os distintos sítios alimentares. Essa situação de elevada intensidade de pastejo (baixa oferta de forragem) foi relatada por Aguinaga (2004), Pinto et al. (2007) e Santos (2007), que observaram que, em pastos manejados com baixa oferta de forragem (4\%), a massa de forragem se manteve em valores limitantes $(779,698$ e $803 \mathrm{~kg} / \mathrm{ha}$ de MS, respectivamente), determinando o baixo desempenho animal, em virtude da quase ausência de oportunidade de seleção. Isso significa que o animal é obrigado a buscar e consumir forragem de maneira pouco seletiva, pois não existe opção de seleção e a composição da dieta do animal se aproxima da média existente no pasto.
Não foi observada diferença entre as ofertas de forragem $(\mathrm{P}>0,1)$, e sim entre faixas $(\mathrm{P}<0,1)$ quanto à frequência de altura e massa de forragem nos sítios alimentares. Houve predomínio de sítios alimentares com altura e massa de forragem limitantes (Tabela 2) à ingestão de forragem pelos animais (Gonçalves, 2007), tendo em vista a amplitude estratégias de manejo de oferta de forragem empregadas. $\mathrm{O}$ maior percentual $(\mathrm{P}<0,1)$ de sítios alimentares foi detectado em alturas inferiores a $6,0 \mathrm{~cm}$ e em massas de forragem de até $1.500 \mathrm{~kg} /$ ha de MS. Ressalta-se, no entanto, que houve redução considerável de $66 \%$ para $34 \%$ dos sítios alimentares na faixa menor que $6 \mathrm{~cm}$ de altura, em comparação às estratégias com oferta de forragem de $12 \%$ e $16-12 \%$, respectivamente. Em massa de forragem, essa redução foi de $34,8 \%$ para $1,5 \%$, o que pode significar modificações no comportamento ingestivo e não no desempenho animal (Parsons, 1994).

Observando a frequência de sítios alimentares em faixa de massa de forragem e altura do pasto nas quais a ingestão de forragem não estaria sendo limitada, como nas faixas 8-12 $\mathrm{cm}$ e 1.500 a $2.500 \mathrm{~kg} / \mathrm{ha}$ de MS, as estratégias de oferta de forragem $8-12 \%$ e $16-12 \%$ foram as que mais criaram sítios alimentares com estruturas favoráveis ao consumo animal. As somas do percentual de sítios alimentares para essas duas faixas são de 26,4 e $47,7 \%$ para ofertas de forragem de $16-12 \%, 27,1 \%$ e $34,8 \%$ para a estratégia de manejo $8-12 \%$, respectiva-mente para altura do pasto e massa de forragem. Nas faixas acima de $12 \mathrm{~cm} \mathrm{e} 2.500 \mathrm{~kg} / \mathrm{ha}$ de MS, o percentual de sítios alimentares atingiu aproximadamente $30 \%$ na oferta de forragem de 16-12\%. Segundo Gonçalves (2007), esses tipos de sítios alimentares também podem limitar a ingestão de forragem, uma vez que, em alturas de pasto acima de $11,4 \mathrm{~cm}$, ocorre maior dispersão das lâminas nos estratos superiores do dossel, cuja redução de densidade de lâminas deprecia o peso do bocado e a velocidade de ingestão de forragem.

Santos (2007), trabalhando em proposta semelhante, avaliou o percentual de sítios alimentares com massa de

Tabela 1 - Massa de forragem, altura do pasto, área efetivamente pastejada, taxa de acúmulo diário de MS, produção de MS e oferta real de forragem de uma pastagem natural submetida a diversas estratégias de manejo da oferta de forragem

\begin{tabular}{|c|c|c|c|c|c|c|}
\hline $\begin{array}{l}\text { Oferta de } \\
\text { forragem } \\
(\% \mathrm{PV})\end{array}$ & $\begin{array}{c}\text { Massa de } \\
\text { forragem } \\
(\mathrm{kg} / \mathrm{ha} \text { de } \mathrm{MS})\end{array}$ & $\begin{array}{l}\text { Altura do } \\
\text { pasto }(\mathrm{cm})\end{array}$ & $\begin{array}{l}\text { Área } \\
\text { efetivamente } \\
\text { pastejada }(\%)\end{array}$ & $\begin{array}{c}\text { Taxa de } \\
\text { acúmulo } \\
\text { diário de } \mathrm{MS} \\
(\mathrm{kg} / \mathrm{ha} \text { de } \mathrm{MS})\end{array}$ & $\begin{array}{c}\text { Produção } \\
\text { de MS }\end{array}$ & $\begin{array}{l}\text { Oferta real } \\
\text { de forragem } \\
(\% \mathrm{PV})\end{array}$ \\
\hline $8 \%$ & $1170 \mathrm{D}(127,4)$ & $5,5 \mathrm{D} \quad(0,58)$ & $76,9 \mathrm{AB} \quad(3,50)$ & $9,21 \quad(3,21)$ & $4088(851,7)$ & $8,4 \mathrm{D} \quad(0,85)$ \\
\hline $12 \%$ & $1720 \mathrm{AB}(128,6)$ & $8,4 \mathrm{ABC}(0,59)$ & $66,8 \mathrm{C} \quad(3,52)$ & $8,39(3,26)$ & $3063(851,7)$ & $12,2 \mathrm{C}(0,92)$ \\
\hline $16 \%$ & $1935 \mathrm{AB} \quad(130,8)$ & $9,3 \mathrm{AB} \quad(0,60)$ & $62,8 \mathrm{C} \quad(3,57)$ & $11,78 \quad(3,29)$ & $4991 \quad(851,7)$ & $16,2 \mathrm{~A} \quad(0,93)$ \\
\hline $8-12 \%$ & $1640 \mathrm{BC}(131,20)$ & $7,6 \mathrm{BC} \quad(0,60)$ & $78,4 \mathrm{~A} \quad(3,59)$ & $14,63(3,31)$ & $5009(851,7)$ & $11,6 \mathrm{BC}(1,02)$ \\
\hline $12-8 \%$ & $1350 \mathrm{CD} \quad(128,9)$ & $6,8 \mathrm{CD} \quad(0,59)$ & $70,1 \mathrm{BC} \quad(3,53)$ & $8,82 \quad(3,25)$ & $2911 \quad(851,7)$ & $9,3 \mathrm{D} \quad(0,92)$ \\
\hline $16-12 \%$ & $2050 \mathrm{~A}(128,4)$ & $9,5 \mathrm{~A} \quad(0,59)$ & $65,1 \mathrm{C}(3,52)$ & $12,3 \quad(3,24)$ & $4347(851,7)$ & $14,3 \mathrm{AB} \quad(0,93)$ \\
\hline
\end{tabular}

A, B, C, D: Médias seguidas de letras diferentes na coluna diferem $(\mathrm{P}<0,10)$ pelo teste $\mathrm{t}$.

Valores entre parênteses representam o erro-padrão da média. 
Tabela 2 - Frequência de sítios alimentares em diferentes faixas de altura do pasto e massa de forragem em uma pastagem natural submetida a diversas estratégias de manejo da oferta de forragem

\begin{tabular}{|c|c|c|c|c|c|}
\hline \multirow[b]{2}{*}{ Oferta de forragem } & \multicolumn{5}{|c|}{ Altura do pasto $(\mathrm{cm})$} \\
\hline & $<6$ & $6-8$ & $8-10$ & $10-12$ & $>12$ \\
\hline $8 \%$ & $58,2 \mathrm{a}$ & $8,0 \mathrm{~b}$ & $14,2 b$ & $6,0 \mathrm{~b}$ & $13,6 b$ \\
\hline $12 \%$ & $66,0 \mathrm{a}$ & $11,8 \mathrm{~b}$ & $12,8 \mathrm{~b}$ & $4,2 b$ & $5,3 \mathrm{~b}$ \\
\hline $16 \%$ & $56,0 \mathrm{a}$ & $10,0 \mathrm{~b}$ & $12,2 \mathrm{~b}$ & $6,8 \mathrm{~b}$ & $15,0 \mathrm{~b}$ \\
\hline $8-12 \%$ & $40,5 \mathrm{a}$ & $11,0 \mathrm{c}$ & $18,9 b$ & $8,2 \mathrm{c}$ & $21,4 \mathrm{~b}$ \\
\hline $12-8 \%$ & $53,0 \mathrm{a}$ & $7,3 \mathrm{~cd}$ & $15,4 \mathrm{~b}$ & $5,5 \mathrm{~b}$ & $18,8 \mathrm{~b}$ \\
\hline \multirow[t]{2}{*}{$16-12 \%$} & $33,9 a$ & $8,4 \mathrm{c}$ & $18,0 \mathrm{~b}$ & $8,4 \mathrm{c}$ & $33,9 \mathrm{a}$ \\
\hline & \multicolumn{5}{|c|}{ Massa de forragem $(\mathrm{kg} / \mathrm{ha}$ de $\mathrm{MS})$} \\
\hline Oferta de forragem & $<1.000$ & $1.000-1.500$ & $1.500-2.000$ & $2.000-2.500$ & $>2.500$ \\
\hline $8 \%$ & $24,8 b$ & $42,9 a$ & $19,5 \mathrm{bc}$ & $4,8 \mathrm{c}$ & $8,0 \mathrm{c}$ \\
\hline $12 \%$ & $34,8 \mathrm{a}$ & $47,2 \mathrm{a}$ & $12,4 \mathrm{~b}$ & $3,1 \mathrm{~b}$ & $2,4 \mathrm{~b}$ \\
\hline $16 \%$ & $23,1 \mathrm{~b}$ & $44,8 \mathrm{a}$ & $15,4 b c$ & $4,9 \mathrm{c}$ & $11,8 b c$ \\
\hline $8-12 \%$ & $15,5 \mathrm{c}$ & $33,3 \mathrm{a}$ & $25,1 \mathrm{~b}$ & $9,8 \mathrm{c}$ & $16,3 b c$ \\
\hline $12-8 \%$ & $27,0 \mathrm{~b}$ & $36,3 \mathrm{a}$ & $15,3 \mathrm{c}$ & $8,1 \mathrm{c}$ & $13,2 \mathrm{c}$ \\
\hline $16-12 \%$ & $1,5 \mathrm{c}$ & $21,3 \mathrm{ab}$ & $34,4 \mathrm{a}$ & $13,3 b c$ & $29,4 \mathrm{a}$ \\
\hline
\end{tabular}

Médias seguidas de letras diferentes na linha diferem $(\mathrm{P}<0,10)$ pelo teste $t$.

forragem ou altura do pasto dentro de faixas consideradas não-limitantes ao consumo de forragem. Com base em resultados de diversos trabalhos de pesquisas, o autor considerou faixas de massa de forragem entre $1.400 \mathrm{e}$ $2.500 \mathrm{~kg} / \mathrm{ha}$ de MS e de altura do pasto entre 7,5 e 13,5 cm como não-limitantes à ingestão diária de MS pelos animais, sugerindo que com modificações no comportamento ingestivo (aumento do tempo de pastejo) os bovinos poderiam compensar uma perda na estrutura mantendo o mesmo nível de ganho.

Considerando sítios alimentares em que a altura e a massa de forragem estariam prejudicando o potencial de ingestão de forragem por serem baixas, como faixas de altura $<6 \mathrm{~cm}$ e $6-8 \mathrm{~cm}$, e em massas de forragem de $<1.000$ e 1.000 $1.500 \mathrm{~kg} / \mathrm{h}$ a de MS, a oferta de forragem de $16-12 \%$ foi a que apresentou menor percentual de sítios alimentares nestas faixas ( $42 \%$ e $23 \%$, respectivamente, para altura e massa de forragem). Santos (2007) constatou que as ofertas de forragem de $16 \%$ e $16-12 \%$ concentraram cerca de $50 \%$ dos sítios alimentares em faixas de massa de forragem de 1.400 a $2.500 \mathrm{~kg} / \mathrm{ha}$ de MS, e $35 \%$ de sítios alimentares em faixas entre 7,5 e 13,5 cm de altura, que foram superiores às demais estratégias de manejo estudadas.

Sítios com faixas maiores que $12 \mathrm{~cm}$ e massas de forragem maiores que $2.500 \mathrm{~kg} / \mathrm{ha}$ de MS podem ser considerados limitantes ao potencial de ingestão dos bovinos (Gonçalves, 2007), porém ambientes com maior percentual de sítios alimentares nesta faixa são mais encontrados nas estratégias de manejo com níveis de oferta de forragem de $16 \%$ e manejos que variam a oferta de forragem na primavera $(8-12 \%, 12-8 \%$ e $16-12 \%)$. Esse tipo de ambiente parece ter um tipo de "reserva" de forragem nessas faixas superiores e, embora não seja a estrutura mais adequada para o animal, em certas situações, como nos períodos de outono/inverno e estiagens, os animais podem vir a utilizar esses sítios.

Os resultados evidenciam a frequência inexpressiva de sítios alimentares em faixas consideradas ótimas à taxa de ingestão por bovinos, mesmo em situações em que se tenha elevada oferta de forragem (Tabela 2). Isso, em parte, explica as razões pelas quais o desempenho individual, apesar de responder positivamente a aumentos na oferta de forragem (Maraschin, 2001), atinge valores baixos em pastagens naturais de duplo estrato quando manejadas exclusivamente com ajuste da taxa de lotação a oferta de forragem. Segundo Parsons (1994), sítios alimentares preferidos, quando ocorrem em frequências menores que $20 \%$, deixam de ser selecionados ativamente pelos animais, pois o dispêndio energético para selecionar esses sítios passa a não compensar o processo, a não ser que estejam agregados, como ocorre caracteristicamente nas áreas de baixadas. Em decorrência disso, como o percentual de sítios alimentares na faixa ótima praticamente não ultrapassa $10 \%$ para altura e $13 \%$ para massa de forragem, o animal acaba exercendo seleção passiva por esses sítios, utilizando-os somente quando os encontra ao acaso durante o dia de pastejo.

O comportamento entre a dinâmica espaço-temporal das faixas de massa de forragem e altura do pasto e as estratégias de manejo da oferta de forragem foi semelhante, porém, a maior concentração de sítios alimentares ocorreu na faixa ALT $<6 \mathrm{~cm}$ (Figura 1A) e houve um equilíbrio entre as faixas $\mathrm{MF}<1.000$ e 1.000-1.500 kg/ha de MS (Figura 1B).

A interação oferta de forragem $\times$ estação do ano foi significativa $(\mathrm{P}<0,1)$ para as faixas de altura $<6 \mathrm{~cm}, 6-8 \mathrm{e}$ 
$8-10 \mathrm{~cm}$ e para faixas de massa de forragem $<1.000$ e 2.000 $2.500 \mathrm{~kg} / \mathrm{ha}$ MS, de modo que suas respectivas probabilidades foram de 0,$0039 ; 0,0596$ e 0,0275 para as faixas de altura, e 0,0195 e 0,0048 para as de massa de forragem, respectivamente. A interação observada reflete o grau de mobilidade das faixas ao longo das estações.

De modo geral, a interação parece ser produto da seletividade animal e indicaria que as faixas intermediárias seriam utilizadas de forma não-aleatória, com uma relação entre o período do ano e a utilização dos sítios alimentares. Por exemplo, o aumento da faixa altura $<6$ e massa de forragem $<1.000$ do verão para o inverno ocorre pela diminuição proporcional dos sítios nas faixas $6-8,8-10$, $10-12$ para altura e $1.000-1.500,1.500-2.000,2.000-2.500$ para massa de forragem ao longo do mesmo período. Aparentemente, os animais utilizam essas faixas prioritariamente em relação à faixa de altura $>12$ e a massa de forragem $>2.500$ enquanto podem, ou até o inverno, quando essa última faixa passa a ser mais utilizada (Figura 1A). A frequência da faixa altura $<6$ e massa de forragem $<1.000$ parece ser consequência da intensidade de uso das demais, pois a utilização de qualquer uma delas tem "efeito dominó" sobre a primeira, ou seja, acaba realocando os sítios explorados em direção a essa faixa mais inferior. A repartição mais homogênea dos sítios alimentares no verão de 2006 pode ser explicada por um diferimento (período de descanso) de 70 dias antes do início do experimento, que fez com que aumentasse a frequência dos sítios alimentares nas faixas intermediárias.

A partir dessa estação, iniciam-se os efeitos das estratégias de manejo e, particularmente durante o outono, cria-se a maior predominância dos sítios alimentares nas faixas inferiores, comportamento similar para a maioria das estratégias de manejo de oferta de forragem adotadas, à exceção da oferta de forragem de 16-12\%, que manteve sempre menor $(\mathrm{P}<0,1)$ frequência dos sítios alimentares na faixa de altura menor que $6 \mathrm{~cm}$ e massa de forragem menor que $1.000 \mathrm{~kg}$ de MS por hectare em qualquer estação do ano. Enquanto em quase todas as ofertas de forragem tiveram aumentos significativos de sítios alimentares nesta faixa inferior já no outono, o mesmo ocorreu somente no período de inverno para o manejo de oferta de forragem de 16-12\%, o que reflete a existência de maior "banco de forragem" disponível nesta estratégia de manejo, que retarda o rebaixamento do pasto e avança até o inverno com pasto em estrutura ainda não limitante.

$\mathrm{O}$ aumento na frequência de sítios alimentares na faixa de altura menor que $6 \mathrm{~cm}$ do verão para o outono parece ter sido proporcionalmente maior nas estratégias com oferta de forragem fixas que naquelas com oferta de forragem variável, e esse aumento deve-se principalmente à diminuição dos sítios alimentares nas faixas intermediárias (6-8, 8-10, 10-12 para ALT e 1.000-1.500, 1.500-2.000, 2.000-2.500 para MF). Neste sentido, as estratégias de manejo com oferta de forragem 8-12 e 16-12\% parecem apresentar proporção de sítios alimentares mais equilibrada, com variações menos extremas entre faixas ao longo do tempo, enquanto nas outras estratégias de manejo, em maior ou menor grau, sempre há uma ou duas faixas que se destacam demasiadamente em relação às demais. Como essas faixas nunca são consideradas ótimas, esse fenômeno pode estar relacionado ao fato de que a estratégia com oferta de forragem $8-12 \%$ produz melhores resultados de produção animal, atestado por Santos (2007) e Pinto et al. (2007).

O inverno foi o período com maior concentração de sítios alimentares nas faixas inferiores de altura e massa de forragem $(<8 \mathrm{~cm} \mathrm{e}<1.500 \mathrm{~kg} /$ ha de MS $)$ e coincidiu justamente com o menor desempenho dos animais (Soares et al., 2005). Isso ocorre porque as condições climáticas, principalmente a temperatura, são desfavoráveis para a produção de forragem, já que as pastagens naturais da Depressão Central do Rio Grande do Sul são compostas principalmente por espécies de ciclo estival (Boldrini, 1997).

A menor frequência de sítios alimentares em faixas de altura do pasto menor que $6 \mathrm{~cm}$ ocorreu nas ofertas variáveis na maioria das estações do ano. A estratégia com oferta de forragem de 16-12\% apresentou menor frequência de sítios com altura e massa de forragem na faixa mais limitante ao consumo $(<6 \mathrm{~cm},<1.000)$ em todas as estações do ano. Praticamente não foram encontrados sítios alimentares nesta faixa em nenhuma das estações do ano. O efeito positivo do uso de ofertas de forragem variáveis não é capaz de reverter à redução percentual em aumento da frequência de sítios alimentares na faixa ótima de altura do pasto (10-12 cm de altura), uma vez que, em todas as estações, os manejos da oferta de forragem não apresentaram diferença de frequência nesta faixa $(\mathrm{P}>0,1)$ e os valores médios foram de $10 \%$.

Nas faixas de massa de forragem (Figura 1B) no verão 2006, com o efeito do diferimento, os sítios se concentraram predominantemente na faixa $1.000-1.500 \mathrm{~kg} / \mathrm{ha}$ de MS para as estratégias de manejo com oferta de forragem fixa. Nas estratégias com ofertas de forragem variáveis, principalmente $8-12 \%$ e 16-12\%, houve melhor distribuição dos sítios alimentares nas faixas de massa de forragem superiores. A estratégia com oferta de forragem 16-12\% nesse período concentrou $25 \%$ dos sítios alimentares na faixa considerada ótima (2.000-2.500 kg/ha de MS), que foi superior $(\mathrm{P}<0,1)$ às demais. No outono, a porcentagem de sítios alimentares na faixa ótima diminuiu para $15 \%$ e se 
A)
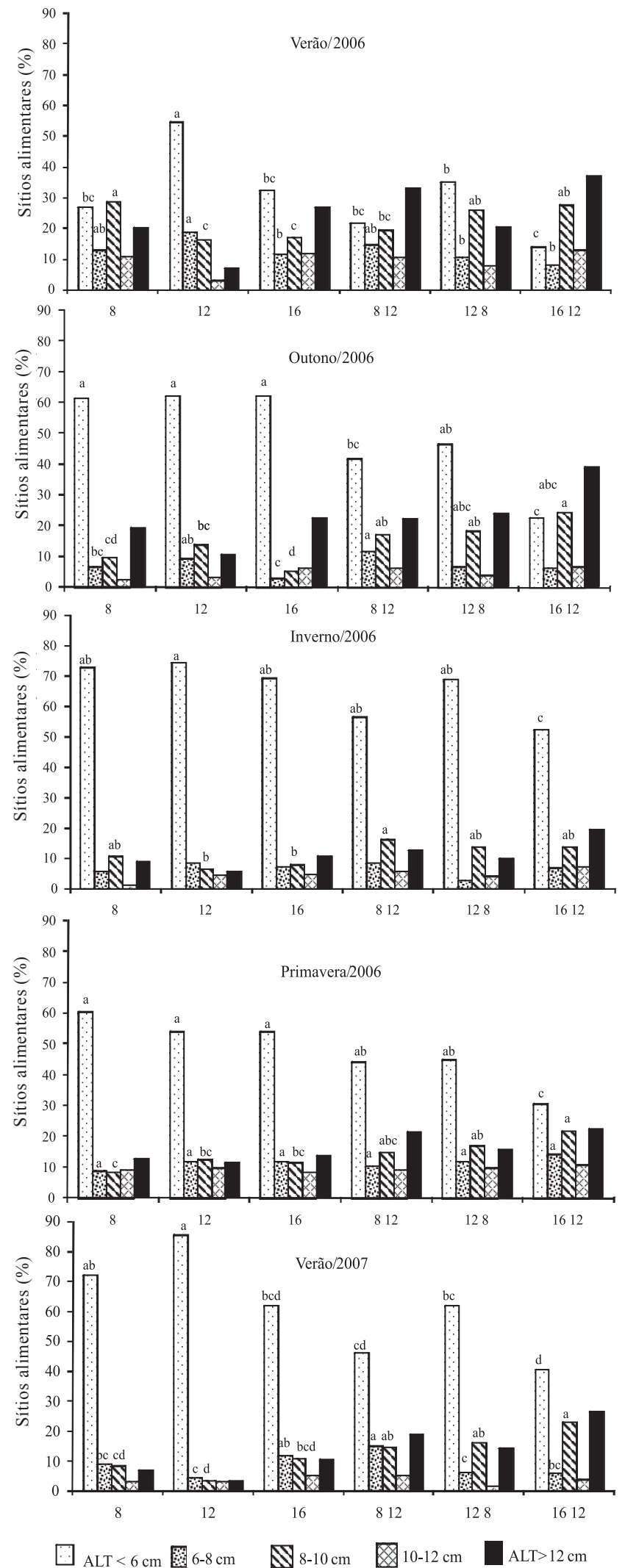

B)

Massa da forragem (kg/ha de MS)
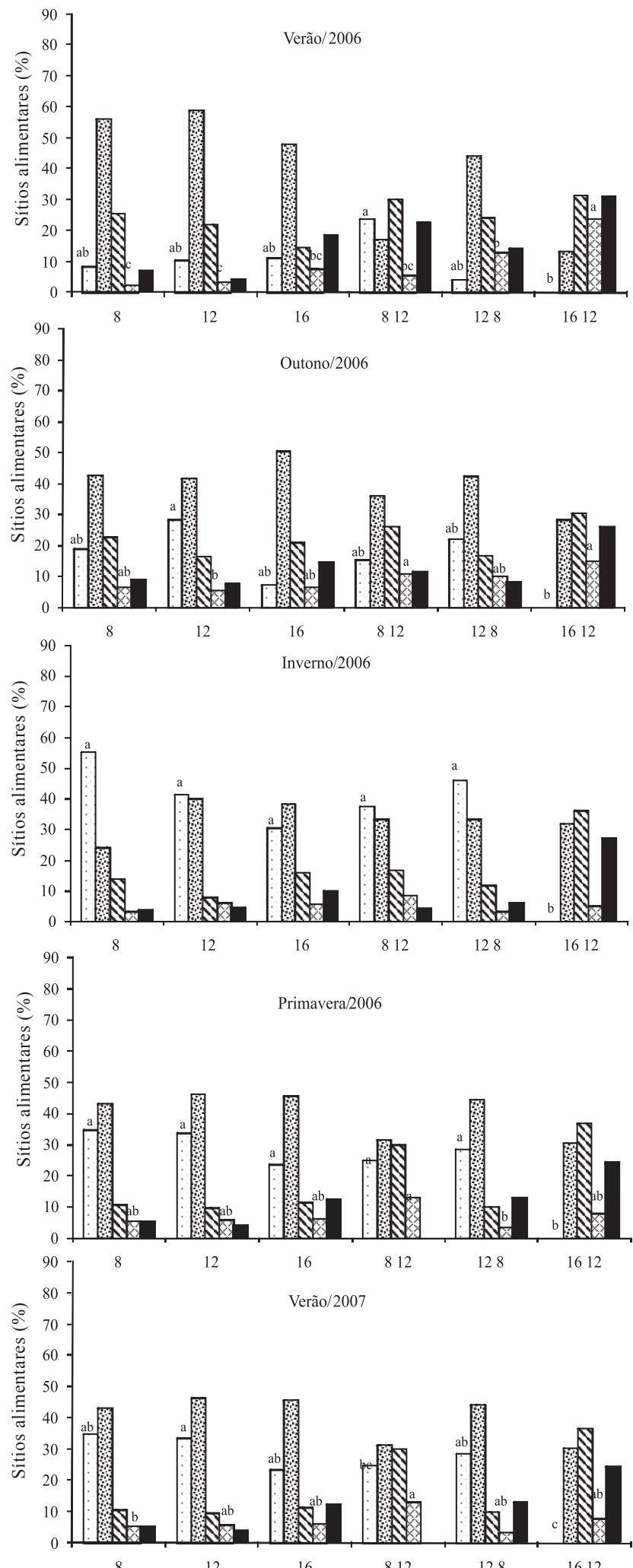

$\square \mathrm{MF}<1000$ 圈1000-1500 1500-2000 圂 2000-2500 MF $>2500$

Médias seguidas de letras distintas, na mesma estação do ano, diferem pelo teste $\mathrm{t}(\mathrm{P}<0,10)$.

Figura 1 - Frequência dos sítios alimentares (\%) em diversas faixas de altura do pasto (A) e massa de forragem (B) em pastagem natural manejada sob diversas estratégias de manejo da oferta de forragem. 
igualou à obtida nas demais estratégias de manejo, com exceção daquela com oferta de forragem de $12 \%$. Nas demais estações, o percentual de sítios alimentares na faixa ótima de massa de forragem variou entre 5 e $10 \%$ e não diferiu entre as estratégias de manejo. Essas duas estratégias de manejo são contrárias, isto é, uma aumenta a oferta de forragem para $16 \%$ na primavera e a outra diminui para $8 \%$ e, mesmo assim, são as que se destacam quanto à adequação de estrutura. No entanto, têm em comum a oferta de forragem de $12 \%$ durante o restante do ano e este é o nível de oferta de forragem que promove os melhores resultados, tanto em produção animal como em produção vegetal ao longo de vários anos de pesquisa (Maraschin, 2001).

\section{Conclusões}

O manejo da oferta de forragem em pastagem natural provoca modificações espaços-temporais na estrutura do campo, em particular sobre a dinâmica da ocupação espacial entre sítios alimentares na área efetivamente pastejada, e também na frequência de espécies e/ou estruturas indesejáveis ao consumo por bovinos. As estratégias de manejo com variações de oferta de forragem na primavera, como $8-12 \%$ e $16-12 \%$, apresentam menor percentual de sítios alimentares em faixas consideradas limitantes ao consumo dos bovinos. Em nenhuma das estratégias de manejo avaliadas, é possível proporcionar a ocorrência de sítios alimentares considerados ótimos ao consumo dos bovinos em pastejo. Para isso, em pastagens de estrutura complexa, é necessária a utilização de outras ferramentas de manejo, além do ajuste da taxa de lotação, a fim de elevar o estrato inferior (efetivamente pastejado) e rebaixar o estrato superior, criando ambientes pastoris com estruturas mais favoráveis à taxa de ingestão de forragem por bovinos e otimizando a colheita da forragem produzida.

\section{Literatura Citada}

AGUinaGA, A.J.Q. Manejo da oferta de forragem e seus efeitos na produção animal e na produtividade primária de uma pastagem natural na Depressão Central do Rio Grande do Sul. 2004. 79f. Dissertação (Mestrado em Zootecnia) Faculdade de Agronomia/Universidade Federal do Rio Grande do Sul, Porto Alegre, 2004.

ARMSTRONG, R.H.; ROBERTSON, E.; HUNTER, E.A. The effect of sward height and its direction of change on the herbage intake, diet selection and performance of weaned lambs grazing ryegrass swards. Grass and Forage Science, v.50, p.389-398, 1995.

BARTHRAM, G.T. Experimental techniques: the HFRO sward stick. In: Hill Farming Research Organization. [S.I.]: n/Biennial Report, 1985, p.29-30, 1985.

BERGAMASCHI, H.; GUADAGNIN, M.R.; CARDOSO, L.S. et al. Clima da Estação Experimental da UFRGS (e Região de Abrangência). Porto Alegre: Universidade Federal do Rio Grande do Sul, 2003. 78p.
BOLDRINI, I.I. Dinâmica da vegetação de uma pastagem natural sob diferentes níveis de oferta de forragem e tipos de solo, Depressão Central, RS. 1993. 262f. Tese (Doutorado em Zootecnia) - Universidade Federal do Rio Grande do Sul, Porto Alegre, 1993

BOLDRINI, I.I. Campos do Rio Grande do Sul: Caracterização fisionômica e problemática ocupacional. Boletim do Instituto de Biociências, n.56, p.1-39, 1997.

BOVAL, M.; FANCHONE, A.; ARCHIME'de, H. et al. Effect of structure of a tropical pasture on ingestive behavior, digestibility of diet and daily intake by grazing cattle. Grass and Forage Science, v.62, p.44-54, 2007.

CARASSAI, I.J.; NABINGER, C.; CARVALHO, P.C.F. et al. Recria de cordeiras em pastagem nativa melhorada submetida à fertilização nitrogenada. 1. Dinâmica da pastagem. Revista Brasileira de Zootecnia, v.37, n. 8, p.1338-1346, 2008.

CARVALHO, P.C.F. A estrutura da pastagem e o comportamento ingestivo de ruminantes em pastejo. In: IMPÓSIO SOBRE AVALIAÇÃO DE PASTAGENS COM ANIMAIS, 1., 1997, Maringá. Anais... Maringá: Universidade Estadual de Maringá, 1997. p.25-52.

CARVALHO, P.C.F.; SANTOS, D.T.; NEVES, F.P. Oferta de forragem como condicionadora da estrutura do pasto e do desempenho animal. In: SIMPÓSIO DE FORRAGEIRAS E PRODUÇÃO ANIMAL: SUSTENTABILIDADE PRODUTIVA DO BIOMA PAMPA, 2., 2007, Porto Alegre. Anais... Porto Alegre: Universidade Federal do Rio Grande do Sul, 2007. p.23-59.

CORREAA, F.L.; MARASCHIN, G.E. Crescimento e desaparecimento de uma pastagem nativa sob diferentes níveis de oferta de forragem. Pesquisa Agropecuária Brasileira, v.29, n.10, p.1617-1623, 1994.

Escosteguy, C.M.D. Avaliação agronômica de uma pastagem natural sob níveis de pressão de pastejo. 1990. 231f. Dissertação (Mestrado em Zootecnia) - Universidade Federal do Rio Grande do Sul, Porto Alegre, 1990.

FONTOURA JR., J.A.S.; CARVALHO, P.C.F.; NABINGER, C. et al Produção de forragem em pastagem submetida ao controle de plantas indesejáveis e a intensidade de pastejo. Ciência Rural, v.37, n.1, p.247-252, 2007.

GONÇALVES, E.N. Comportamento ingestivo de bovinos e ovinos em pastagem natural da Depressão Central do Rio Grande do Sul. 2007. 131f. Tese (Doutorado em Zootecnia) Universidade Federal do Rio Grande do Sul, Porto Alegre, 2007.

LACA, E.A.; DEMMENT, M.W. Herbivory: the dilemma of foraging in a spatially heterogeneous food environment. In: PALO, R.T.; ROBBINS, C.T. (Eds.) Plant defenses against mammalian herbivory. Boca Raton: CRC, 1991. p.29-44.

MARASCHIN, G.E. Production potential of South America grasslands. In: INTERNATIONAL GRASSLAND CONGRESS, 19., 2001 , Piracicaba. Proceedings... Piracicaba: Escola Superior de Agricultura "Luiz de Queiroz", 2001. p.5-18.

MOOJEN, E.L. Dinâmica e potencial produtivo de uma pastagem nativa do Rio Grande do Sul submetida a pressões de pastejo, épocas de diferimento e níveis de adubação. 1991. 172f. Tese (Doutorado em Zootecnia) - Universidade Federal do Rio Grande do Sul, Porto Alegre, 1991.

MORAES, A.; MOOJEN, E.L.; MARASCHIN, G.E. Comparação de métodos de estimativa de taxa de crescimento em uma pastagem submetida a diferentes pressões de pastejo. In: REUNIÃO ANUAL DA SOCIEDADE BRASILEIRA DE ZOOTECNIA, 27. 1990, Campinas. Anais... Piracicaba: Fundação de Estudos Agrários Luiz de Queiroz, 1990. p.332.

PALLARÉS, R.O.; BERRETTA, E.J.; MARASCHIN, G.E. The South American Campos Ecosystem. In: SUTTIE, J.M.; REYNOLDS, S.G.; BATELLO, C. (Eds.) Grasslands of the world. Rome: FAO, 2005. p.171-179.

PARSONS, A.J. A mechanistic model of some physical determinants of intake rate and diet selection in a two species temperate grassland sward. Functional Ecology, v.8, p.187-204, 1994. 
PEDREIRA, C.G.S. Avanços metodológicos na avaliação de pastagens. In: REUNIÃO ANUAL DA SOCIEDADE BRASILEIRA DE ZOOTECNIA, 39., 2002, Recife. Anais... Recife: Sociedade Brasileira de Zootecnia, 2002. p.100-150.

PINTO, C.E.; CARVALHO, P.C.F.; FRIZZO, A. et al. Comportamento ingestivo de novilhos em uma pastagem nativa do Rio Grande do Sul. Revista Brasileira de Zootecnia, v.36, n.2, p.319-327, 2007.

POPPI, D.P.; HUGHES, T.P.; 1'HUILLIER, P.J. Intake of pasture by grazing ruminants. In: NICOL, A.M. (Ed.) Livestock feeding on pasture. Hamilton: New Zealand Society of Animal Production, 1987. p.55-64. (Occasional Publication, 10).

SANTOS, D.T. Manipulação da oferta de forragem em pastagem natural: efeito sobre o ambiente de pastejo e o desenvolvimento de novilhas de corte. 2007. 259f. Tese (Doutorado em Zootecnia) - Universidade Federal do Rio Grande do Sul, Porto Alegre, 2007.

SANTOS, D.T.; CARVALHO, P.C.F.; FREITAS, F.K. et al. Adubação de pastagem natural no Sul do Brasil: 1. Efeito do Nitrogênio sobre a produção primária. In: GRASSLAND ECOPHYSIOLOGY
AND GRAZING ECOlOGY, 2., 2004, Curitiba. Proceedings... Curitiba: Universidade Federal do Paraná, [2004]. (CD-ROM).

SOARES, A.B. Efeito da dinâmica da oferta de forragem sobre a produção animal e de forragem em pastagem natural. 2002. 197f. Tese (Doutorado em Zootecnia) - Universidade Federal do Rio Grande do Sul, Porto Alegre, 2002.

SOARES, A.B.; CARVALHO, P.C.F.; NABINGER, C. et al. Produção animal e de forragem em pastagem nativa submetida a distintas ofertas de forragem. Ciência Rural, v.35, n.5, p.1148-1154, 2005.

STATISTICAL ANALYSIS SYSTEM - SAS. SAS/STAT user's guide: statistics. 4.ed. Version 8.2. Cary: 2001, v.2. 943p.

STUTH, J.W. Foraging behavior. In: HEITSCHMIDT, R. K.; STUTH, J.W. (Eds.) Grazing management: an ecological perspective. Oregon: Timber Press, 1991. p.85-108.

WILM, H.G.; COSTELLO, D.F.; KLIPPLE, G.E. Estimating forage yield by the double sampling method. Journal of American Society of Agronomy, v.36, p.194-203. 1944.

WOLFINGER, R. Covariance structure selection in general mixed models. Communication in Statistics, v.22, p.1079-1106, 1993. 